\title{
Proximal Versus Distal Splenic Artery Embolisation for Blunt Splenic Trauma: What is the Impact on Splenic Immune Function?
}

\author{
P. T. Foley ${ }^{1} \cdot$ H. Kavnoudias ${ }^{2} \cdot$ P. U. Cameron ${ }^{3,4} \cdot$ C. Czarnecki ${ }^{5} \cdot$ \\ E. Paul ${ }^{6}$ - S. M. Lyon ${ }^{7,8}$
}

Received: 11 May 2015 / Accepted: 4 June 2015/Published online: 3 July 2015

(C) Springer Science+Business Media New York and the Cardiovascular and Interventional Radiological Society of Europe (CIRSE) 2015

\begin{abstract}
Purpose To compare the impact of proximal or distal splenic artery embolisation versus that of splenectomy on splenic immune function as measured by $\operatorname{IgM}$ memory $\mathrm{B}$ cell levels.

Materials and Methods Patients with splenic trauma who were treated by splenic artery embolisation (SAE) were enrolled. After 6 months splenic volume was assessed by $\mathrm{CT}$, and IgM memory B cells in peripheral blood were measured and compared to a local normal reference population and to a post-splenectomy population.

Results Of the 71 patients who underwent embolisation, 38 underwent proximal embolisation, 11 underwent distal embolisation, 22 patients were excluded, 1 had both proximal and distal embolisation, 5 did not survive and 16
\end{abstract}

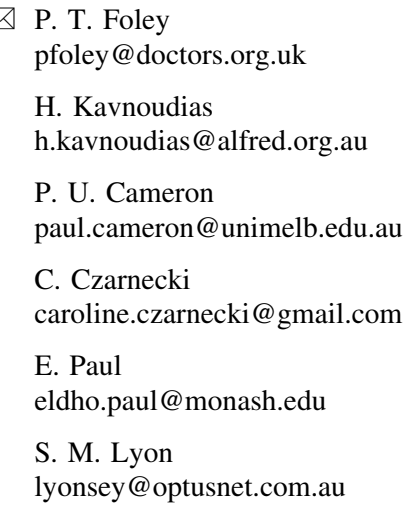

1 Department of Medical Imaging, The Canberra Hospital, Yamba Drive, Garran, ACT 2605, Australia

2 Radiology Research Unit, Radiology Department, The Alfred Hospital, Commercial Rd, Melbourne, VIC 3004, Australia

3 Infectious Diseases Unit, The Alfred Hospital, Commercial Rd, Melbourne, VIC 3004, Australia did not return for evaluation. There was a significant difference between splenectomy and proximal or distal embolisation and a trend towards greater preservation of IgM memory B cell number in those with distal embolisation-a difference that could not be attributed to differences in age, grade of injury or residual splenic volume. Conclusion IgM memory B cell levels are significantly higher in those treated with SAE compared to splenectomy. Our data provide evidence that splenic embolisation should reduce immunological complications of spleen trauma and suggest that distal embolisation may maintain better function.

Keywords Splenic artery embolisation - Trauma . IgM memory B cells

4 Department of Microbiology and Immunology, Doherty Institute for Infection and Immunity, University of Melbourne, 792 Elizabeth St, Melbourne, VIC 3000, Australia

5 Radiology Department, Royal Melbourne Hospital, 300 Grattan St, Parkville, VIC 3050, Australia

6 Department of Epidemiology \& Preventive Medicine, School of Public Health and Preventive Medicine, Alfred Hospital, Monash University, Commercial Rd, Melbourne, VIC 3004, Australia

7 Melbourne Endovascular, 5 Chesterville Rd, Cheltenham, VIC 3192, Australia

8 Radiology Department, The Alfred Hospital, Commercial Rd, Melbourne, VIC 3004, Australia 


\section{Introduction}

Splenic artery embolisation (SAE) has been shown to increase the rate of splenic conservation following trauma, [1-6] and is frequently performed as an adjunct to nonoperative management of blunt splenic injury which is now the standard of care in haemodynamically stable patients [7-10].

There is as yet very limited evidence that SAE preserves splenic immune function. Until the last few years, the immune function of the spleen has not been sufficiently well understood to allow its direct evaluation.

Recently IgM memory B cells have been shown to play a role in the innate response to encapsulated bacteria through $\mathrm{T}$ cell-independent production of IgM antibodies. IgM memory B cells depend on the spleen for development and are reduced in number in the peripheral blood of splenectomised patients. A lack of IgM memory B cells is associated with increased vulnerability of post-splenectomy patients to pneumococcal infections.

Two methods of splenic artery embolisation have been described: proximal embolisation to occlude the splenic artery proximal to the spleen, reducing perfusion pressure but allowing splenic perfusion via collaterals; distal embolisation to occlude the bleeding vessel within the spleen, usually resulting in infarction of the tissue distal to the embolisation but with normal perfusion pressure to the remaining spleen. The effects of these different methods on splenic immune function have not been previously evaluated.

The aim of this study was to compare the effects of proximal splenic artery embolisation, distal splenic artery embolisation and splenectomy on splenic immune function, using the level of IgM Memory B cells as a marker.

\section{Materials and Methods}

This study took place over a 6-year period between February 2004 and October 2010. Trauma patients identified as having splenic injuries on contrast-enhanced multidetector computed tomography (CE-MDCT) and fulfilling our criteria, (Table 1), underwent SAE and were prospectively enrolled in this study.

Patients who were haemodynamically unstable or who had other indications for laparotomy were treated surgically.

The procedures were performed by the on-call interventional radiologist. Proximal embolisation was defined as occlusion of the main splenic artery distal to the dorsal pancreatic artery. Distal embolisations were defined as occlusion of a branch distal to the main splenic artery. The choice of embolic material and of proximal or distal embolisation was up to the preference of the interventionalist, taking into account the anatomy of the injury.
Table 1 Guidelines for splenic artery embolisation for blunt splenic injury at The Alfred

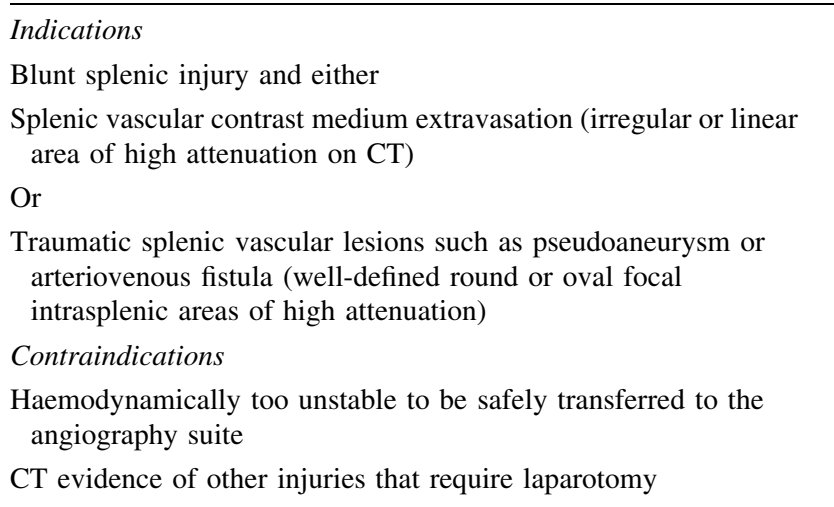

After the embolisation procedure, the Victorian Splenic Registry was notified and organised immunisations for these patients.

These patients were then rescanned after at least 6 months to assess splenic volume post embolisation. The splenic volumes on the follow-up CT scans were calculated using volume rendering software (GE Healthcare, Milwaukee, WI, USA).

The initial grade of splenic injury of all patients was retrospectively assessed according to the method described by Mirvis et al. [11] (Table 2) by at least two board-certified radiologists who were blinded to the method of embolisation used. Differences of opinion were resolved by discussion and consensus after re-examination of the study.

The patients were also followed up with a blood test performed at least 6 months after embolisation to assess total IgM memory B cell level, percentage of IgM memory B cells within the B cell population, presence of Howell Jolly bodies and platelet levels. The methods used to assess IgM memory B cells have been described previously [12]. The levels of IgM memory B cells were then compared to the reference ranges previously established by the laboratory directed by one of the authors (PUC). The control group was selected from normal blood bank donors and

Table 2 CT-based injury severity grades and criteria for blunt splenic trauma

Grade I: Capsular avulsion, laceration(s), or subcapsular haematoma $<1 \mathrm{~cm}$ diameter

Grade II: Laceration(s) 1-3 cm deep, central/subcapsular haematoma $1-3 \mathrm{~cm}$ diameter

Grade III: Laceration(s) 3-10 cm deep, central/subcapsular haematoma $>3 \mathrm{~cm}$ diameter

Grade IV: Laceration(s) $>10 \mathrm{~cm}$ deep, central/subcapsular haematoma $>10 \mathrm{~cm}$ diameter, massive lobar maceration or devascularisation

Grade V: Bilobar tissue maceration or devitalisation

From Mirvis et al. 11 
represented a range of ages as previously described [12]. They were not age or sex matched to the donors.

The study did not alter the medical management of the patient, requiring only an additional blood test and CT at 6 months, and was approved by the ethics committee of this institution before enrolment commenced.

Statistical analysis was performed using SAS software version 9.2 (SAS Institute, Cary, NC. USA). IgM memory $\mathrm{B}$ cell levels were assessed for normality and found to have an approximate normal distribution after logarithmic transformation. Comparisons between groups were performed using analysis of variance or Mann-Whitney $U$ test where appropriate, with results reported as geometric mean (95\% confidence interval) or median (inter-quartile range). Post-hoc comparisons were performed using Bonferroni adjustment for multiple comparisons. A two-sided $P$ value of 0.05 was considered statistically significant.

\section{Results}

Patient numbers and classification are shown in Table 3 and demographic details in Table 4. Out of a total of 71 patients who underwent splenic artery embolisation for trauma during the study period, $\mathbf{3 8}$ patients who underwent proximal embolisation and $\mathbf{1 1}$ who underwent distal embolisation were included in the study. 1 patient was excluded as they underwent both proximal and distal embolisation. 5 patients were excluded as they did not survive to 6 months follow-up. One of these died of fat embolism syndrome during their acute admission; two others survived to discharge but died subsequently-one was known to have mesothelioma. In two cases, the records were missing so the cause of death could not be determined.

11 patients were lost to follow-up as they were uncontactable. Three patients were contacted but refused to

Table 3 Breakdown of patients included in and excluded from the study

\begin{tabular}{lr}
\hline Included & \\
Proximal embolisation & 38 \\
Distal embolisation & 11 \\
Excluded & 1 \\
Proximal and distal embolisation & 5 \\
Deceased before follow-up & 11 \\
Lost to follow-up & 3 \\
Uncontactable & 2 \\
Refused & \\
Found not to have undergone blood tests & \\
\hline
\end{tabular}

return for follow-up. Two patients were excluded as they had not had IgM memory B cell tests.

There were 5 females in the proximal embolisation group and one in the distal embolisation group; all other patients were male.

The different groups were compared in terms of $\operatorname{IgM}$ Memory B cell numbers, using IgM Memory B cells as a percentage of total $\mathrm{B}$ cells as the metric. The results are displayed in Table 5. They demonstrate a significant difference between both the proximal and distal groups compared to the splenectomy group. A significant difference between distal and proximal groups was not found; however, there was a significant difference between the control group and the proximal but not the distal group.

The proximal and distal groups were compared in terms of residual splenic volumes and grades of injury to see if there were significant differences which could account for the differences in terms of IgM Memory B cells at followup. The results are displayed in Table 6 .

As can be seen, we demonstrated no significant difference between the two groups in terms of age, grade of injury, or residual splenic volume at follow-up.

\section{Discussion}

Traumatic injury of the spleen was historically routinely treated with splenectomy. This leads to loss of immunity to encapsulated organisms, in particular streptococcus pneumoniae, and the risk of overwhelming post-splenectomy infection (OPSI) [13] with a mortality rate of 50-70\%. [14]. Moreover splenectomy is associated with other disadvantages including an increased risk of premature death [15].

Today, splenic conservation is preferred with surgery reserved for patients who are haemodynamically unstable or who have other injuries requiring laparotomy. The object of SAE is to improve the rate of splenic conservation in the nonsurgical management group. However, there is very limited evidence that SAE preserves splenic immune function. It is only in recent years that the immune function of the spleen has become better understood and useful assays have become available to evaluate splenic immune function directly.

The immune function of the spleen includes a specific role in the defence against encapsulated bacteria. Production of natural antibody and innate immunity against bacterial infection has been attributed to B1 cells and to IgM Memory B cells which are found in the splenic marginal zone (SMZ) as well as in the peripheral blood, tonsils, and lymph nodes [16, 17]. SMZ B cells require a functional spleen for optimal development and maintenance. The SMZ surrounds the white pulp of the spleen and is farthest 


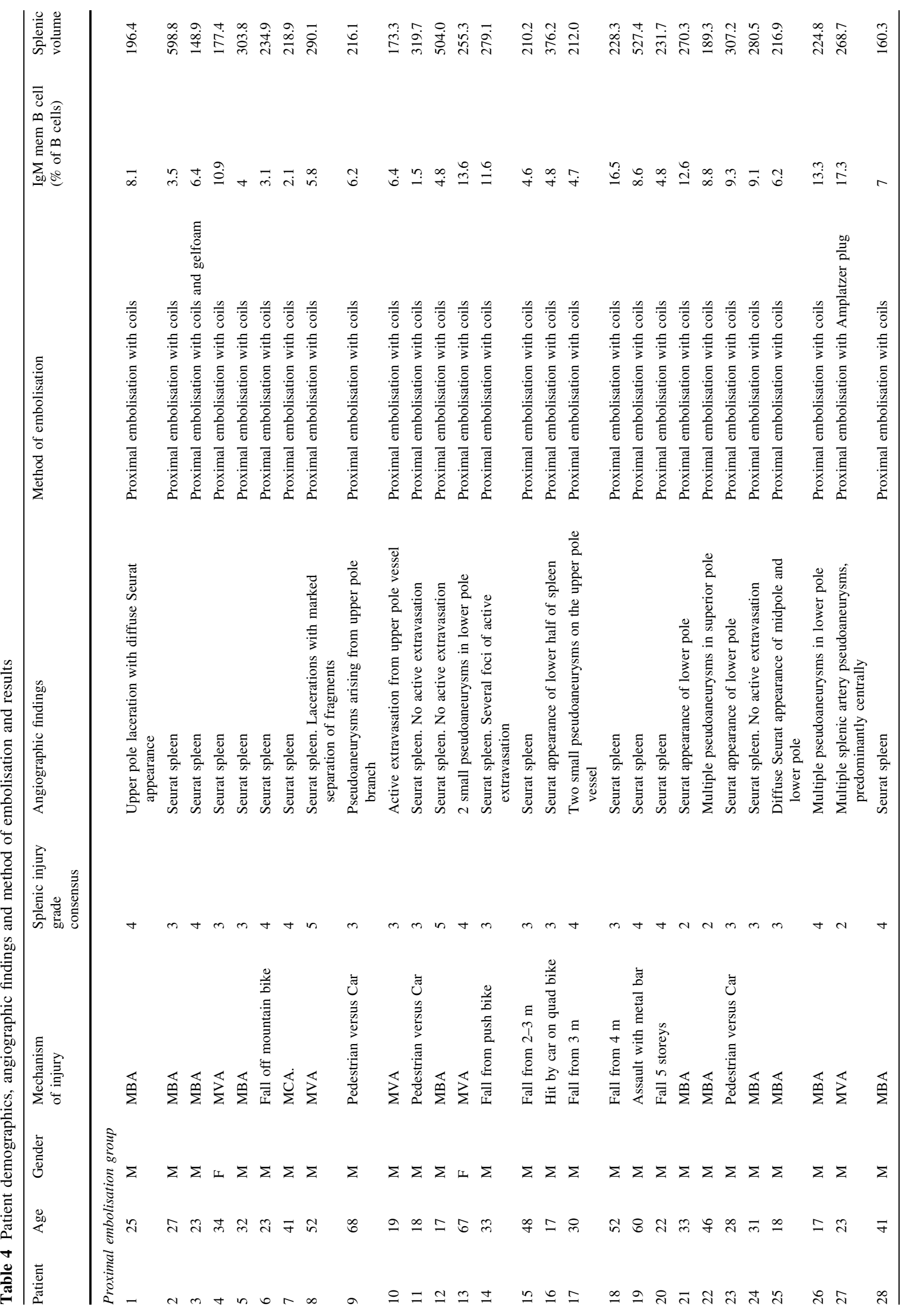




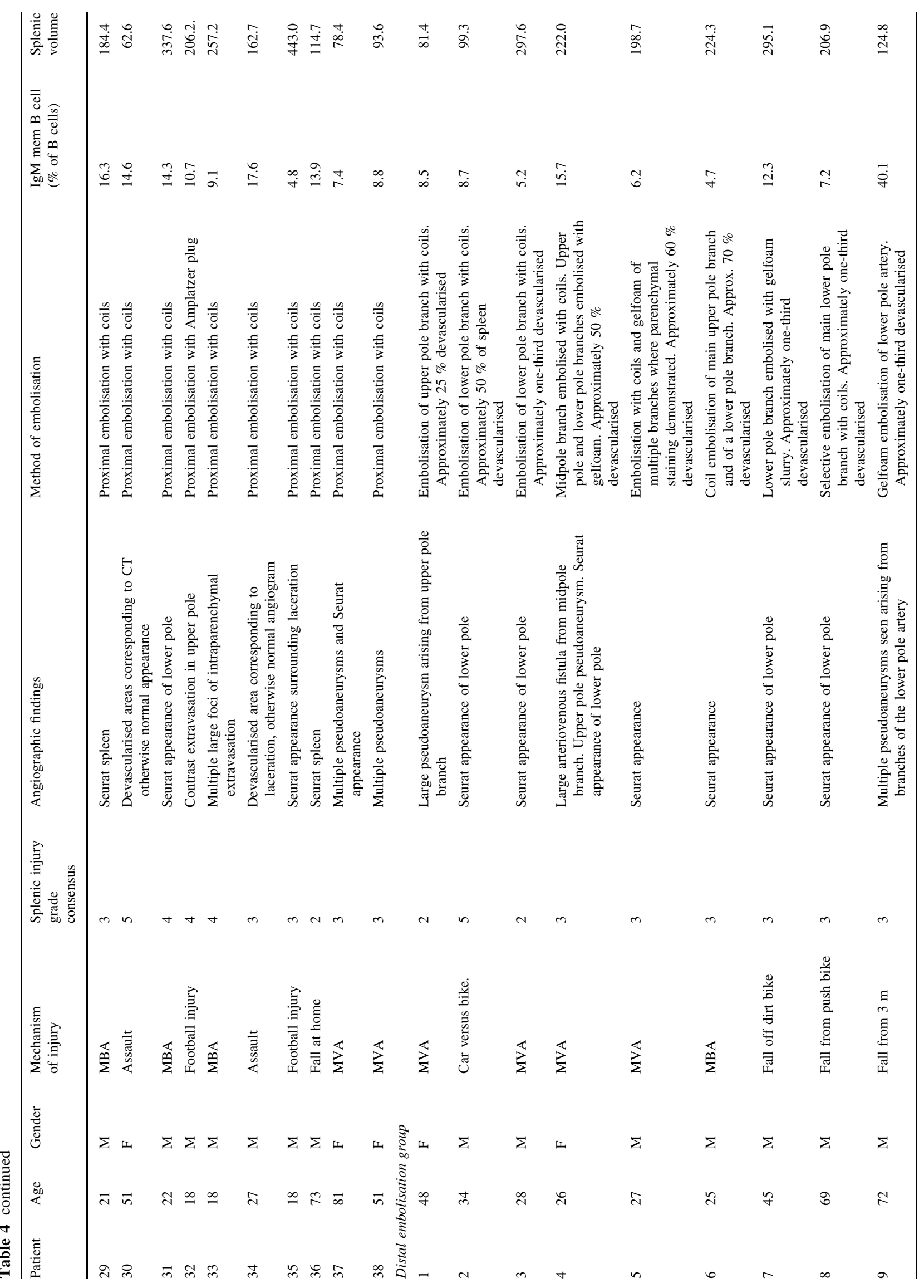


away from the central arteriole. It may, therefore, represent a watershed zone which is more vulnerable than other zones to a fall in splenic perfusion pressure.

Around half of the B cells in the blood express the CD27 marker and are considered memory B cells $[18,19]$. There are two populations of memory B cells-switched memory B cells and IgM memory B cells. Switched memory B cells produce high-affinity antibodies and protect against reinfection [20].

The role of IgM memory B cells is to respond rapidly to blood-borne polysaccharide antigens expressed by encapsulated bacteria. Upon stimulation, the prediversified IgM memory B cells initiate a T-cell-independent response, rapidly proliferating and differentiating into antigen-presenting cells or into IgM-, IgG- and IgA-secreting plasma cells. Unlike switched memory B cells, IgM memory B cells do not promote long-term protective humoral immune responses but give protection for approximately six months [21]. Deficiency or absence of IgM memory B cells is associated with increased frequency of pneumococcal infections and impaired response to polysaccharide pneumococcal vaccines [16].

IgM memory B cell levels are reduced or absent in splenectomised and asplenic individuals [12, 16, 20, 21]. A significant reduction or absence of IgM memory B cells is also found in patient groups with an intact spleen but known increased risk of invasive pneumococcal infection [18].

The level of circulating IgM memory B cells can therefore be considered to be a marker for splenic immune function, and for the degree of protection against infection and OPSI.

It is important to note that the spleen plays a role in preventing OPSI over and above the role of the SMZ environment for memory B cells. It entraps circulating pathogens during systemic infections. OPSI never occurs in individuals lacking IgM memory B cells but with an intact spleen, such as young children and some CVID patients [19]. This strengthens the case for preserving the spleen by embolisation.

Proximal SAE (PSAE) is performed by occluding the splenic artery just distal to the origin of the dorsal pancreatic artery. The aim of PSAE is to decrease splenic perfusion pressure and thereby increase the likelihood of haemostasis whilst preserving some perfusion of the spleen via collateral vessels [10]. It has been shown to reduce splenic intra-arterial blood pressure by $>50 \%$, albeit in a study involving only 2 patients [22].

Distal embolisation is often used for the treatment of focal vascular injuries such as pseudoaneurysms or arteriovenous fistulae [4, 23]. Subsequent bleeding may occur because some vascular injuries are initially not detected at angiography or CT due to vasospasm [4, 23]. However, a 
Table 5 IgM Memory B cells as percentage of total B cells in the different groups

\begin{tabular}{lclc}
\hline Group & Geometric mean $(95 \%$ CI) (IgM memory & Post-hoc comparisons & $\begin{array}{l}\text { Adjusted } \\
P \text { value }\end{array}$ \\
\hline Control & B cells as \% of total B cells) & Control versus distal & 1.00 \\
Distal & $10.75(9.58-12.06)$ & Control vs proximal & 0.02 \\
Proximal & $9.97(6.75-14.75)$ & Control versus splenectomy & $<0.0001$ \\
Splenectomy & $7.55(6.11-9.31)$ & Distal versus proximal & 1.00 \\
& $2.97(2.54-3.48)$ & Distal versus splenectomy & $<0.0001$ \\
& & Proximal versus splenectomy & $<0.0001$ \\
\hline
\end{tabular}

Table 6 Comparison between distal and proximal groups

\begin{tabular}{lccc}
\hline Variable & \multicolumn{1}{l}{ Group } & & $P$ value \\
\cline { 2 - 3 } & Distal $(n=11)$ & Proximal $(n=38)$ & \\
\hline Age (years) & $34(26-48)$ & $27.5(19-48)$ & 0.60 \\
Splenic volume (mls) & $206.9(124.8-295.1)$ & $228.3(184.4-290.1)$ & 0.25 \\
Splenic injury grade & $3(3-3)$ & $3(3-4)$ & 0.27 \\
\hline
\end{tabular}

Values are median (inter-quartile range) unless stated otherwise. Wilcoxon rank-sum test was used to compare age, splenic volume and splenic injury grade between groups recent meta-analysis found that the only difference in short-term outcomes between the two techniques was a higher rate of splenic infarcts after distal embolisation [10].

As it is currently utilised in the management of blunt splenic trauma, the effect of SAE on splenic function has not been well defined. One other group has reported that IgM memory B cell levels are preserved after SAE [24]. Their study only included 15 patients and they did not specify whether embolisation was proximal or distal.

Other groups have attempted to assess the effects of SAE on splenic immune function using previously available methods.

Bessoud and co-workers reported preservation of antibody responses to polysaccharide antigens in a series of 24 trauma patients who underwent proximal vessel SAE [25]. At 6-63 months after SAE, all of the patients had sufficient exposure-driven immunity against Haemophilus influenzae type b; $94 \%$ had sufficient exposure-driven immunity against Pneumococcus and $83 \%$ had a sufficient response to Pneumococcus vaccination.

Tominaga et al. [26] compared levels of CD3, CD4, CD8 cells, and IgG, IgM, C3 and Compliment Factor B between patients who had undergone combined (proximal plus distal) SAE, controls and splenectomy patients. They found that CD8 levels were significantly higher in the splenectomy group, whilst still within the normal range, but otherwise there were no differences between the three groups.

Neither of these studies, however, was able to measure splenic immune function directly. Our study, complementing that of Skattum et al. [24], has provided direct evidence of the impact of SAE on splenic immune function, and has provided evidence that both proximal and distal embolisation preserve splenic immune function by comparison with splenectomy.

We had hypothesised that distal embolisation would better preserve splenic function than proximal embolisation, since proximal embolisation leads to a global fall in splenic arterial perfusion pressure potentially putting all the splenic marginal zones at risk of infarction, whereas with distal embolisation only the embolised portions of the spleen are affected. Our results did not show a significant difference in IgM Memory B cells between the two groups, but did show a significant difference between the control group and the proximal but not the distal embolisation group. We conclude from this that there was a trend towards significance but that the numbers in the distal embolisation group were too small to prove this.

However both groups maintained IgM Memory B cell levels significantly higher than those in the splenectomy group, confirming that SAE by either method preserves splenic immune function better than splenectomy. In the absence of long-term follow-up data on infections from the splenic registry, it is however not yet possible to say whether or not these patients still require pneumococcal vaccination and antibiotic prophylaxis.

Our study has limitations and our numbers are too small to draw definite conclusions. A significant number of patients were lost to follow-up. Although patients were prospectively enrolled in the study, they were not randomised, and the choice of proximal versus distal embolisation was up to the individual operator. This 
introduces potential bias, and future studies should randomise patients with injuries where there is equipoise between the two methods. We were obliged to grade splenic injury using the method of Mirvis et al. [13] which does not take into account the degree of vascular injury, rather than the more recent modification of Marmery and Shanmuganathan [8], which differs subtly in that it incorporates splenic vascular injury as Grade IVa. This was because the thin-slice CT data were not archived to the picture archiving and communication system (PACS) in every case.

The SMZs are likely to be the most vulnerable to hypotension as described above. We were unable to control for the degree of hypotension due to blood loss experienced by patients, which is likely to have been most severe prior to the onset of treatment. It may be that hypovolaemic shock can cause damage to the marginal zones even in the absence of splenic trauma. The question of whether global hypotension contributes to hypoperfusion of the SMZs and subsequent loss of IgM Memory B cells could only be answered by an animal study.

In conclusion, our study provides further evidence to support the concept that splenic artery embolisation allows preservation of splenic immune function, and suggests that distal embolisation may better preserve immune function than proximal embolisation. Further research will be necessary to evaluate this hypothesis.

Acknowledgments The authors would like to acknowledge the help of Dr Marcus Pianta in evaluating CTs of splenic injury. We acknowledge Penelope Jones and the staff of the Victorian spleen registry for providing management of vaccination, patient education and long-term follow-up of patients.

\section{Compliance with Ethics Standards}

Conflict of interest There are no relevant conflicts of interest.

Human and Animal Rights and Informed Consent All procedures performed in studies involving human participants were in accordance with the ethical standards of the institutional and/or national research committee and with the 1964 Helsinki declaration and its later amendments or comparable ethical standards. Informed consent was not obtained from patients. This was because the study involved retrospective data collection and did not alter the medical management of the patients.

\section{References}

1. Haan J, Ilahi ON, Kramer M, Scalea TM, Myers J. Protocoldriven nonoperative management in patients with blunt splenic trauma and minimal associated injury decreases length of stay. J Trauma. 2003;55:317-21.

2. Liu PP, Lee WC, Cheng YF, Hsieh PM, Hsieh YM, Tan BL, et al. Use of splenic artery embolization as an adjunct to nonsurgical management of blunt splenic injury. J Trauma. 2004;56:768-72.
3. Sclafani SJ, Shaftan GW, Scalea TM, Patterson LA, Kohl L, Kantor A, et al. Nonoperative salvage of computed tomographydiagnosed splenic injuries: utilization of angiography for triage and embolization for hemostasis. J Trauma. 1995;39:818-25.

4. Haan JM, Biffl W, Knudson MM, Davis KA, Oka T, Majercik S, et al. Splenic embolization revisited: a multicenter review. J Trauma. 2004;56:542-7.

5. Haan J, Scott J, Boyd-Kranis RL, Ho S, Kramer M, Scalea TM. Admission angiography for blunt splenic injury: advantages and pitfalls. J Trauma. 2001;51:1161-5.

6. Hagiwara A, Yukioka T, Ohta S, Nitatori T, Matsuda H, Shimazaki S. Nonsurgical management of patients with blunt splenic injury: efficacy of transcatheter arterial embolization. AJR Am J Roentgenol. 1996;167:159-66.

7. Dent D, Alsabrook G, Erickson BA, Myers J, Wholey M, Stewart $\mathrm{R}$, et al. Blunt splenic injuries: high nonoperative management rate can be achieved with selective embolization. J Trauma. 2004;56(5):1063-7.

8. Marmery H, Shanmuganathan K. Multidetector-row computed tomography imaging of splenic trauma. Semin Ultrasound CT MR. 2006;27(5):404-19.

9. Ekeh AP, McCarthy MC, Woods RJ, Haley E. Complications arising from splenic embolization after blunt splenic trauma. Am J Surg. 2005;189(3):335-9.

10. Schnüriger B, Inaba K, Konstantinidis A, Lustenberger T, Chan LS, Demetriades D. Outcomes of proximal versus distal splenic artery embolization after trauma: a systematic review and metaanalysis. J Trauma. 2011;70(1):252-60.

11. Mirvis SE, Whitley NO, Gens DR. Blunt splenic trauma in adults: CT-based classification and correlation with prognosis and treatment. Radiology. 1989;171:33-139.

12. Cameron PU, Jones P, Gorniak M, Dunster K, Paul E, Lewin S, Woolley I, Spelman D. Splenectomy associated changes in IgM memory B cells in an adult spleen registry cohort. PLoS One. 2011;6(8):e23164.

13. de Porto AP, Lammers AJ, Bennink RJ, ten Berge IJ, Speelman $\mathrm{P}$, Hoekstra JB. Assessment of splenic function. Eur J Clin Microbiol Infect Dis. 2010;29(12):1465-73.

14. Richardson JD. Changes in the management of injuries to the liver and spleen. J Am Coll Surg. 2005;200(5):648-69.

15. Crary SE, Buchanan GR. Vascular complications after splenectomy for hematologic disorders. Blood. 2009;114(14):2861-8.

16. Carsetti R, Rosado MM, Wardmann H. Peripheral development of B cells in mouse and man. Immunol Rev. 2004;197:179-91.

17. Mebius RE, Kraal G. Structure and function of the spleen. Nat Rev Immunol. 2005;5(8):606-16.

18. Di Sabatino A, Rosado MM, Ciccocioppo R, Cazzola P, Morera R, Corazza GR, Carsetti R. Depletion of immunoglobulin M memory $B$ cells is associated with splenic hypofunction in inflammatory bowel disease. Am J Gastroenterol. 2005;100(8):1788-95.

19. Kruetzmann S, Rosado MM, Weber H, Germing U, Tournilhac $\mathrm{O}$, Peter $\mathrm{HH}$, et al. Human immunoglobulin M memory B cells controlling Streptococcus pneumoniae infections are generated in the spleen. J Exp Med. 2003;197(7):939-45.

20. Wasserstrom H, Bussel J, Lim LCL, Cunningham-Rundles C. Memory B cells and pneumococcal antibody after splenectomy. J Immunol. 2008;181(5):3684-9.

21. Weller S, Faili A, Garcia C, et al. CD40-CD40L independent Ig gene hypermutation suggests a second $\mathrm{B}$ cell diversification pathway in humans. Proc Natl Acad Sci USA. 2001;98:1166-70.

22. Bessoud B, Denys A. Main splenic artery embolization using coils in blunt splenic injuries: effects on the intrasplenic blood pressure. Eur Radiol. 2004;14:1718-9.

23. Davis KA, Fabian TC, Croce MA, et al. Improved success in nonoperative management of blunt splenic injuries: embolisation of splenic artery pseudoaneurysms. J Trauma. 1998;44:1008-13. 
24. Skattum J, Loekke RJ, Titze TL, Bechensteen AG, Aaberge IS, Osnes LT, et al. Preserved function after angioembolisation of splenic injury in children and adolescents: a case control study. Injury. 2014;45(1):156-9.

25. Bessoud B, Duchosal MA, Siegrist CA, Schlegel S, Doenz F, Calmes JM, et al. Proximal splenic artery embolization for blunt splenic injury: clinical, immunologic, and ultrasound-Doppler follow-up. J Trauma. 2007;62(6):1481-6.

26. Tominaga GT, Simon FJ Jr, Dandan IS, Schaffer KB, Kraus JF, Kan M, et al. Immunologic function after splenic embolization, is there a difference? J Trauma. 2009;67(2):289-95. 